\title{
Sensory alien hand syndrome: case report and review of the literature
}

\author{
Hakan Ay, Ferdinando S Buonanno, Bruce H Price, Dean A Le, Walter J Koroshetz
}

\begin{abstract}
An 81 year old right handed woman developed a left alien hand syndrome characterised by involuntary movements of choking and hitting the face, neck, and shoulder. The patient showed multiple disorders of primary sensation, sensory processing, hemispatial attention, and visual association, as well as a combination of sensory, optic, and cerebellar ataxia (triple ataxia) of the left arm in the absence of motor neglect or hemiparesis. Imaging studies disclosed subacute infarction in the right thalamus, hippocampus, inferior temporal lobes, splenium of corpus callosum, and occipital lobe due to right posterior cerebral artery occlusion. This rare syndrome should be considered as a "sensory" or "posterior" form of the alien hand syndrome, to be distinguished from the "motor" or "anterior" form described more commonly.

(F Neurol Neurosurg Psychiatry 1998;65:366-369)
\end{abstract}

Department of

Neurology, Stroke

Service, VBK-802

Massachusetts General

Hospital, Harvard

Medical School,

32 Fruit Street, Boston

MA 02114, USA

$\mathrm{H}$ Ay

F S Buonanno

D A Le

W J Koroshetz

Department of

Neurology, McLean

Hospital,

115 Mill Street,

Belmont, MA

02178-9106, USA

B H Price

Correspondence to:

Dr Ferdinando S Buonanno,

Department of Neurology,

Stroke Service, VBK-802

Massachusetts General

Hospital, 32 Fruit Street,

Boston MA, 02114, USA

Telephone 001617726

84 59; fax 00161772650

43.

Received 8 September 1997 and in revised form

6 January 1998

Accepted 14 January 1998
Keywords: alien hand syndrome; posterior; ischaemic stroke; posterior cerebral artery

Although several studies have reported on patients with alien hand syndrome since Goldstein's initial description, ${ }^{1}$ all but three were due to frontal or anterior callosal lesions. ${ }^{2-5}$ Here we describe the clinical, neuroanatomical, and PET findings in a patient with alien hand syndrome due to multiple disorders of primary sensation and sensory processing after right posterior cerebral artery infarction.

\section{Case report}

An 81 year old right handed woman, with an unremarkable medical history, developed spells of left face, arm, and leg tingling. On admission elsewhere, she was confused and disoriented to place; she misidentified her daughter as a nurse; and had left sided numbness, mild weakness, and clumsiness of the arm, spatial neglect, and a homonymous hemianopsia. On the second day, she complained that her left hand was acting as if under someone else's control; it hit her face and head and she reported that she was afraid of it.

On transfer to the Massachusetts General Hospital, she occasionally scratched her left face and stroked her left forehead with her left hand. She made no attempt to look at her hand to correct the movements. She held her left hand with the right, claiming to keep "him" from hitting her. When asked what trouble brought her to the hospital, she said that her "left hand tried to strangle her". She repeated that "someone" was hitting and choking her neck, face, and shoulder. She asked the nurse to restrain her left hand, fearful that "hitting of the breast will cause cancer". These intermittent movements had an irregular speed with slow and smooth onset but became more jerky and rapid before hitting her body.

On examination, she was alert, with right gaze preference, severe left dysmetria with side to side swaying and dysdiadocochinesia, hemispatial neglect, hemianopia, and was anosognosic of any deficits. She moved less often on the left but was not hemiparetic. She displayed severe misreaching with either arm. She showed the right hand when asked to show the left and visually could not distinguish her left hand from the examiner's. Her left hand could not correctly imitate postures imposed on the right hand. Her ability to copy simple diagrams with eyes open was characterised by a series of irregular pen strokes; constructions were somewhat better when performed with her eyes closed. She could not recognise the faces of well known persons from photographs. When shown an advertisement with two children in a car, she saw only the rightward child and did not perceive the car. Visual identification of colours and objects were normal. Her attempts to visually fix on objects in the room to command were delayed and inaccurate. There was severe primary sensory loss on the left side. She extinguished to double simultaneous stimuli over the left side.

On the fifth hospital day, she was still afraid of her left hand despite cessation of the abnormal movements. She placed a pillow on her chest for protection. Her misreaching with either hand and ability to fixate visually improved. Left arm dysmetria was less prominent. Anosognosia, sensory deficits, left hemispatial neglect, and the left visual field cut remained unchanged. She continued to exhibit intermittent disorientation to place; talked to herself as if she was working at home, misidentified her hospital room as her home library or kitchen, and was unable to point to the window and door as directed. By the end of the second 


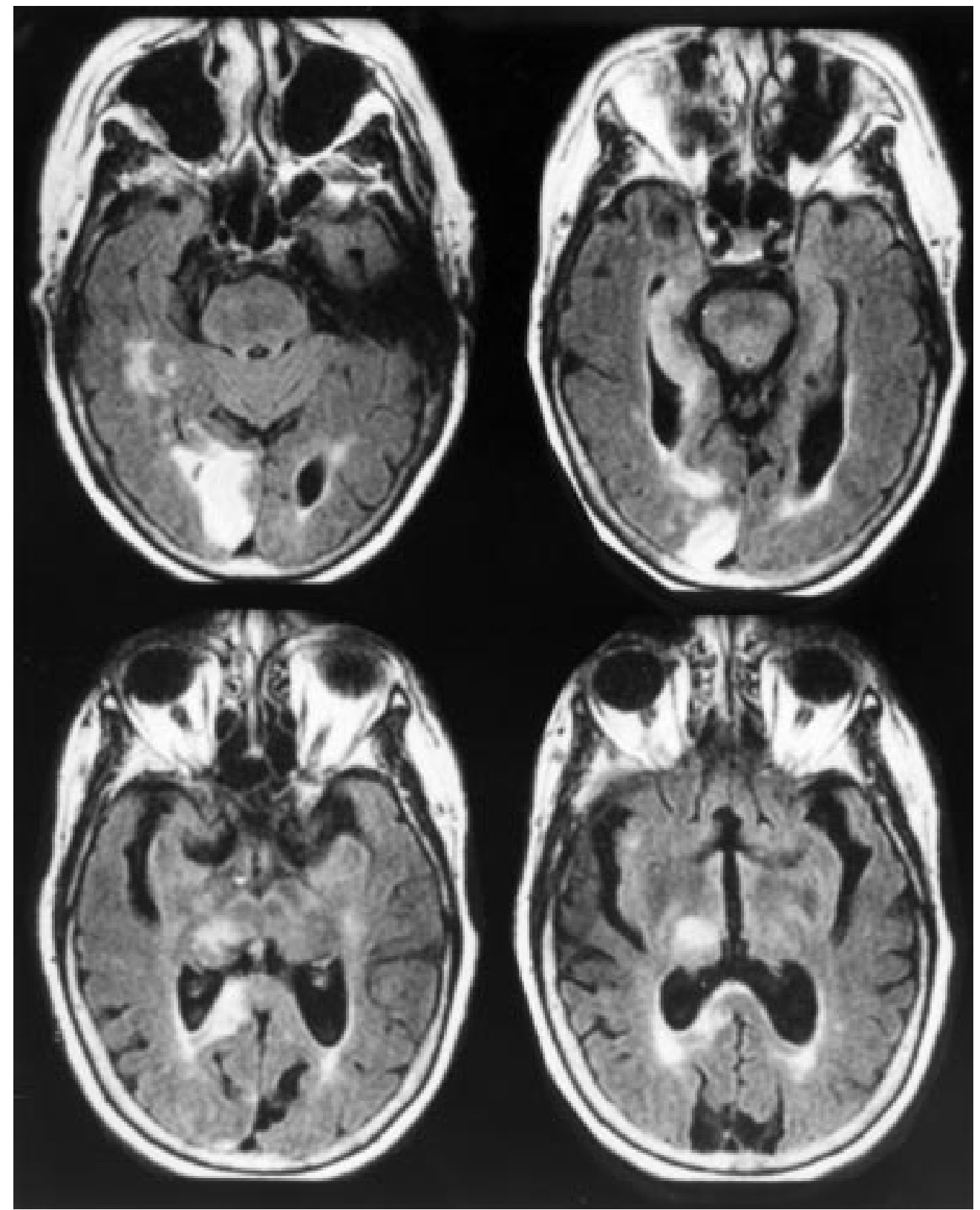

Figure 1 Axial FLAIR-MR images (TR:10002, TE:141/Ef) show high signal indicating a subacute infarction in the right thalamus, hippocampus, inferior-medial temporal lobes, splenium of the corpus callosum, and occipital lobe (from top right to bottom left in ascending order).

hospital week, she no longer feared her left hand. Her appendicular ataxia, visuo-spatial orientation, and left hemineglect improved. Primary sensory deficits remained.

Brain MRI at admission showed a subacute infarction in the right thalamus, hippocampus, inferior temporal lobes, splenium of the corpus callosum, and occipital lobe (fig 1). Two dimensional phase contrast magnetic resonance angiography visualised the proximal $\mathrm{P}-1$ but not distal P-1 or P-2 segments of the right posterior cerebral artery. A definite cause for the posterior cerebral artery occlusion was not uncovered, although a polymerase chain reaction based test disclosed that she was heterozygous for factor V Leiden mutation. Ten days after her admission, a brain PET study was performed using $5.3 \mathrm{mCi}{ }^{18} \mathrm{~F}$-fluorodeoxyglucose (FDG). It showed focally decreased
FDG uptake in the regions known to be infarcted (fig 2); in addition, there was a subtle decrease of FDG uptake in the right posterior frontoparietotemporal regions.

\section{Discussion}

Alien hand syndrome is defined as unwilled, uncontrollable, but seemingly purposeful movements of an upper limb. Two major criteria for the diagnosis are complaint of a foreign limb and complex, autonomous, involuntary motor activity that is not part of an identifiable movement disorder. A verbally expressed feeling that the movements are not under self control and personification of the arm, also occur. ${ }^{6}$ According to this definition, our patient fulfills the criteria for alien hand syndrome.

Our patient differs substantially from those described in the literature. A recent review of 


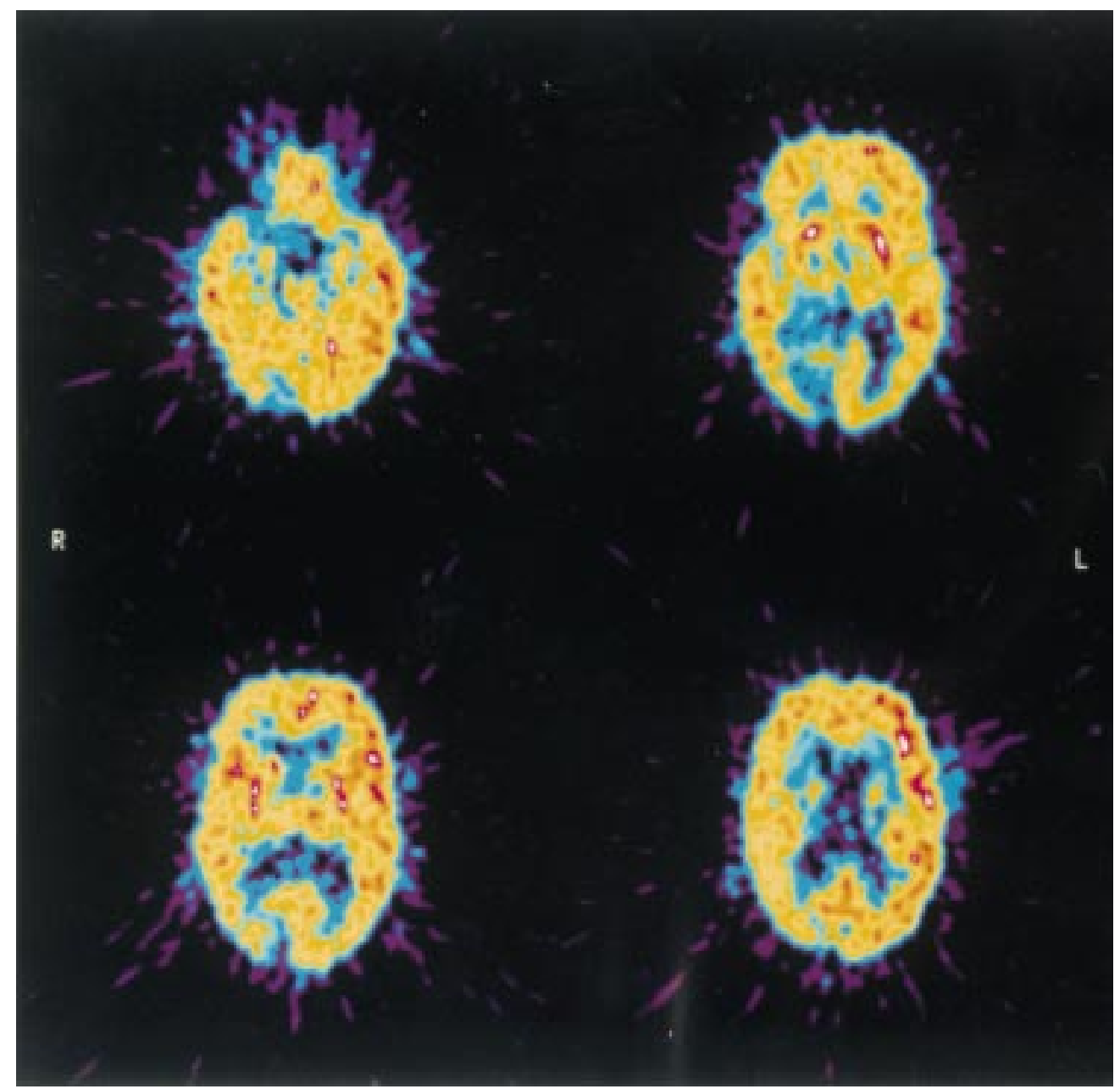

Figure 2 Brain PET images $\left({ }^{8} \mathrm{FDG} ; 6 \mathrm{~mm}\right.$ thick sections reconstructed at $8 \mathrm{~mm}$ resolution FWHM; GE Medical Systems 4096 tomograph) show regions of decreased uptake in the right occipital lobe, the right thalamus, and the right inferior mesial temporal lobe. There was also subtle but widespread decrease in uptake in the right hemispheric cortex. (Courtesy of $S$ Weise, MGH PET Centre.)

20 cases showed that all but one of the responsible cerebral lesions were frontal and always involved the anterior corpus callosum, usually with additional involvement of the supplementary motor area, anterior cingulum, and medial prefrontal cortex. ${ }^{7}$ In the classic syndrome, the main symptom is the report of alienness with abnormal movements of the hand; persistent groping, compulsive manipulation of tools, intermanual conflict, and restraining actions of the normal limb. ${ }^{6} 8$

As opposed to the frontal/callosal alien hand cases in which motor plans are abnormally activated and callosal pathology prevents normal transfer to the opposite hemisphere, our patient had a complicated sensory disorder with self stimulation from the neglected side. A similar clinical syndrome due to posterior cerebral artery infarction was reported by Levine and $\mathrm{Rinn}^{9}$ in a right handed woman with a homonymous hemianopia, hemianaesthesia, and opticosensory ataxia due to proximal right PCA infarction. These authors first suggested that alien hand movements might represent exaggerated or poorly controlled spontaneous movements in the presence of opticosensory ataxia. The report of Doody and Jancovic ${ }^{10}$ included a patient with a large right posterior infarct involving the corpus callosum and posterior internal capsule sparing the thalamus. The exact arterial territory involved was not specified. There were left hemisensory deficits and hemiinattention. The patient thought that the hand with coarse involuntary movements had hostile motivations. Ventura et $a l^{11}$ described a patient with a right thalamomesencephalic haemorrhage, moderate hemisensory deficits, and movements that were interpreted as imitation synkineses; also, the patient presented spontaneous left arm movements perceived as a levitation "as if someone else was moving the arm"; there was no anosognosia, nor hemiasomatognosia; a PET scan 7 weeks after admission showed right frontoparietotemporal cortical hypometabolism, most pronounced in the sensorimotor area. However, it is not only the misperception of abnormal movement that defines alien hand syndrome, but what makes it unique is the patient's subjective acknowledgement that the limb is "not self", and the self destructive or autoaggressive nature of the movements.

One major element in the posterior alien hand syndrome is self stimulation by the left arm. Limb ataxia was severe in both our patient and the patient of Levine and Rinn'. Sensory (ventroposterolateral thalamic infarction) and 
cerebellar (interruption of dentatorubrothalamic tract projection to the ventrolateral thalamus ${ }^{12}$ ) ataxia was worsened by ineffective visual compensation due to the optic ataxia (splenium and occipital cortex infarction). The combination of sensory, optic, and cerebellar ataxia (triple ataxia) in our patient might have caused extremely uncoordinated self stimulatory arm and hand movements ipsilateral to the dense hemianopia and visual and sensory neglect.

Complicated combinations of more than one movement disorder may be seen after thalamic lesions. ${ }^{13}$ The episodic, paroxysmal, and transient nature of the involuntary left arm movements in the "posterior" alien hand cases suggest an extrapyramidal origin. However, the self hitting and self choking movements resemble normal motor plans; in our patient, they may have occurred due to misguided, spontaneous, automatic arm movements associated with emotional expressions (talking, anger, gesturing, stress, scratching the face, stroking the forehead) and postural adjustments, which-in the presence of triple ataxia - could lead to self hitting.

Why were these movements perceived as alien? In addition to misidentifying the source of her own tactile stimulation, our patient made other errors in identification and causation. She misidentified persons and her location, and feared minor breast trauma as a cause of cancer. She also had disordered visual association, being unable to identify faces of famous persons or objects in her room. Her lesion, involving right occipital cortex and splenium, disconnected the right parietal cortex from either occipital lobe, analogous to a left sided lesion causing alexia without agraphia. Moreover, due to hemispatial neglect and optic apraxia there was complete ignorance of the moving left arm. Impaired visuospatial performance and hemineglect are not rare in thalamic infarctions, especially in the territory of the non-dominant tuberothalamic artery ${ }^{14}$ or more laterally placed lesions. ${ }^{15}$ A PET study performed when the left hemineglect was still present but improved and the threatening alien hand movements had stopped, showed altered metabolism in the right frontotemporoparietal regions, possibly indicating a remote effect of the thalamic lesion ("diaschisis"). Most patients with right posterior cerebral artery infarctions do not exhibit an alien hand sign. In stroke syndromes, the combination of sensory neglect and spontaneous self stimulation by the neglected extremity is rare; lesions which cause visuospatial neglect, hemianopia, limb ataxia, and sensory loss often also cause hemiparesis or motor neglect, which preclude stimulation from the neglected side.

Our patient together with the only other previously reported case of Levine and Rinn ${ }^{9}$ argue that left sided alien hand syndrome can be seen as a distinct syndrome in infarctions of the non-dominant posterior cerebral artery territory. It results from a complex combination of triple ataxia, hemianopia, left hemineglect, and primary sensory loss in the absence of motor neglect or hemiparesis, This syndrome should be considered a "sensory" or "posterior" form of the alien hand syndrome, as distinguished from the more commonly described "motor" or "anterior" form.

1 Goldstein K. Zur Lehre von der motorischen Apraxie. Fournal fur Psychologie und Neurologie 1908;11:169-86.

2 Goldberg G, Mayer MH, Toglia JU. Medial frontal cortex infarction and the alien hand sign. Arch Neurol 1981;38: 683-6.

3 McNabb AW, Carroll WM, Mastaglia FL. Alien hand and loss of bimanual coordination after dominant anterior cerebral artery territory infarction. 7 Neurol Neurosurg Psychiatry 1988;1:218-22.

4 Banks G, Short P, Martinez AJ, et al. The alien hand syndrome. Arch Neurol 1989;46:456-9.

5 Riley DE, Lang AE, Lewis A, et al. Cortico-basal ganglionic Riley DE, Lang AE, Lewis A, et al. Cortico-bas
degeneration. Neurology 1990;40:1203-12.

6 Bogen JE. The callosal syndrome. In: Heilman KM, Valen-

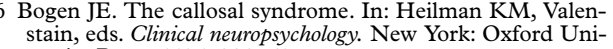
versity Press, 1979:308-59.

7 Feinberg TE, Schindler RJ, Flanagan NG, et al. Two alien hand syndromes. Neurology 1992;42:19-24.

8 Adie WJ, Critchley M. Forced grasping and grooping. Brain 1927;50:142-76.

9 Levine DN, Rinn WE. Opticosensory ataxia and alien hand syndrome after posterior cerebral artery territory infarcsyndrome after posterior cerebral
tion. Neurology 1986;36:1094-7.

10 Doody RS, Jankovic J. The alien hand and related signs. $\mathcal{F}$ Neurol Neurosurg Psychiatry 1992;55:806-10.

11 Ventura MG, Goldman S, Hildebrand J. Alien hand syndrome without a corpus callosum lesion. $\mathcal{F}$ Neurol Neurosurg Psychiatry 1995;58:735-7.

12 Caplan LR, Dewitt LD, Pessin MS, et al. Lateral thalamic infarcts. Arch Neurol 1988;45:959-64.

13 Lee MS, Marsden CD. Movement disorders following lesions of the thalamus or subthalamic region. Mov Disord 1994;9:493-507.

14 Bogousslavsky J, Regli F, Assal G. The syndrome of unilateral tuberothalamic artery territory infarction. Stroke 1986; 17:434-41.

15 Rafal RD, Postner MI. Deficits in human visual spatial attention following thalamic lesions. Proc Natl Acad Sci attention following tha 